\title{
Control of the local magnetic states in graphene with voltage and gating
}

Gao, Fei; Zhang, Yu; He, Lin; Gao, Shiwu; Brandbyge, Mads

Published in:

Physical Review B

Link to article, DOI:

10.1103/PhysRevB.103.L241402

Publication date:

2021

Document Version

Publisher's PDF, also known as Version of record

Link back to DTU Orbit

Citation (APA):

Gao, F., Zhang, Y., He, L., Gao, S., \& Brandbyge, M. (2021). Control of the local magnetic states in graphene with voltage and gating. Physical Review B, 103(24), [L241402]. https://doi.org/10.1103/PhysRevB.103.L241402

\section{General rights}

Copyright and moral rights for the publications made accessible in the public portal are retained by the authors and/or other copyright owners and it is a condition of accessing publications that users recognise and abide by the legal requirements associated with these rights.

- Users may download and print one copy of any publication from the public portal for the purpose of private study or research.

- You may not further distribute the material or use it for any profit-making activity or commercial gain

- You may freely distribute the URL identifying the publication in the public portal

If you believe that this document breaches copyright please contact us providing details, and we will remove access to the work immediately and investigate your claim 


\title{
Control of the local magnetic states in graphene with voltage and gating
}

\author{
Fei Gao $\odot,{ }^{1}$ Yu Zhang, ${ }^{2}$ Lin He, ${ }^{2}$ Shiwu Gao $\odot,{ }^{3}$ and Mads Brandbyge $\odot{ }^{4}$ \\ ${ }^{1}$ Department of Physics, Technical University of Denmark, DK-2800 Kongens Lyngby, Denmark \\ ${ }^{2}$ Center for Advanced Quantum Studies, Department of Physics, Beijing Normal University, 100875 Beijing, China \\ ${ }^{3}$ Beijing Computational Science Research Center, 100193 Beijing, China \\ ${ }^{4}$ Center for Nanostructured Graphene, Department of Physics, Technical University of Denmark, DK-2800 Kongens Lyngby, Denmark
}

(Received 2 December 2020; revised 17 May 2021; accepted 19 May 2021; published 1 June 2021)

\begin{abstract}
Magnetism of graphene can be created by atomic defects, either hydrogen adsorption or single-carbon vacancy formation, owing to the unpaired $\pi$ electrons around the defects. Here we explore, based on rigorous first principles calculations, the possibility of voltage manipulation of two such types of $\pi$ magnetism in graphene via a scanning tunneling microscope tip. We find a remarkably different behavior. For the hydrogen, the magnetic moment can be switched on and off with voltage-induced doping, whereas, for the carbon vacancy, the spin splitting of the $\pi$ bands persists, almost independent of the extent of doping, due to the coupling between the $\pi$ and the $\sigma$ bonds. Furthermore, the local atomic structures near the vacancy can be reversibly manipulated by a coordination mechanism between an intermediate tip-defect distance and a moderate tip voltage, consequently leading to the reversal of spin polarization of the $\pi$ bands. Voltage control of the local magnetic states may open a new avenue for potential applications in spintronics.
\end{abstract}

DOI: 10.1103/PhysRevB.103.L241402

Voltage control of magnetism at the atomic scale is one of the grand challenges in spintronics and has the potential to bring about revolutionary new spintronic devices down to subnanometer scale [1-6]. New materials with controllable magnetic transitions remain an elusive goal and graphene has attracted attention due to its remarkable electron mobility and weak spin-orbit interaction [7-10]. It is known that graphene can become magnetic by hydrogen adsorption [11], or vacancy formation $[12,13]$, which introduces defect states in the $\pi$ band through its unpaired $p_{z}$ orbitals [14-16]. These defects states are localized in space and show preference to one sublattice, according to Lieb's theorem [17], and can offer a unique candidate for atomic-scale magnetic operation and qubits.

The manipulation of individual $\mathrm{H}$ atoms has been proposed to control the spin states by using a scanning tunneling microscopy (STM) tip [11]. The $\pi$ magnetism of monovacancy graphene is tunable by either nanomechanical distortion within the plane $[18,19]$ or by vertical manipulation with a STM tip [20]. Despite the success in nanomechanical control, voltage control of localized spin states, essential to fast spintronic operation, has not been investigated for graphene. Moreover, it remains unclear how the $\mathrm{H}$ adsorbates and carbon vacancies (CVs) respond to the electric perturbation.

At first glance it seems that $\mathrm{H}$ adsorption defects and CVs in graphene are equivalent with respect to the $\pi$ magnetism. However, recent experiments have shown that the spin splitting of the $\pi$ peaks near the Fermi level of monovacancy graphene is stable, even when the two $\pi$ states are doubly occupied or unoccupied [13]. This behavior is different from the $\mathrm{H}$ case, where a transition is observed from the two $\pi$ peaks to one $\pi$ peak under doping or gating [11].
Understanding the intrinsic differences between $\mathrm{H}-$ and $\mathrm{CV}$ induced local magnetism is of fundamental interest and may provide a clue to spin manipulation in a reversible way for the purpose of spin storage and processing.

Here we investigate the voltage control of the $\pi$ magnetism at $\mathrm{H}$ and $\mathrm{CV}$ local spins by charge doping and explore the feasibility of local manipulation by the local field in a junction between a STM tip and the defect. We find that, for the $\mathrm{H}$ case, the voltage can completely switch the magnetism on and off with a moderate bias $(<1 \mathrm{~V})$, while for the $\mathrm{CV}$ case, the total spin cannot be switched off due to the persistence of the unsaturated $\sigma$ defect state within the graphene plane. Interestingly, two magnetic states, associated with the reversal of the spin of the $\pi$ bands of CV state, can still be manipulated by the tip bias and the tip-sample distance. Voltage control of the local graphene magnetism via a STM tip, as first demonstrated here, opens a new avenue for potential applications in spintronics.

The SIESTA/TRANSSIESTA code was employed for the spin polarized calculations with the GGA-PBE [21] and vdWoptB88 [22] functional for exchange-correlation, and a DZP basis set [23-25]. The energy cutoff of $400 \mathrm{Ry}$ was used to define the real-space grid for numerical integration of electron density. Six transverse $k$ points were used in the transport calculation. All the calculations were carried out with a low electronic temperature of $50 \mathrm{~K}$. The computational parameters used in the equilibrium calculations were checked carefully for convergence and reproduced accurately the results and trends obtained by Vienna ab initio simulation package (VASP) [26]. Physical quantities like density of states, transmission, current, and spin density were extracted by using SISL [27].

We first consider the effect of charge doping on the local moments. Without doping, the magnetic moment upon $\mathrm{H}$ 
(a)

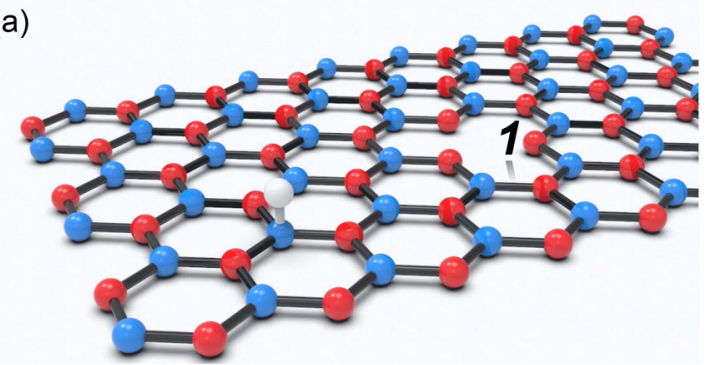

(b)

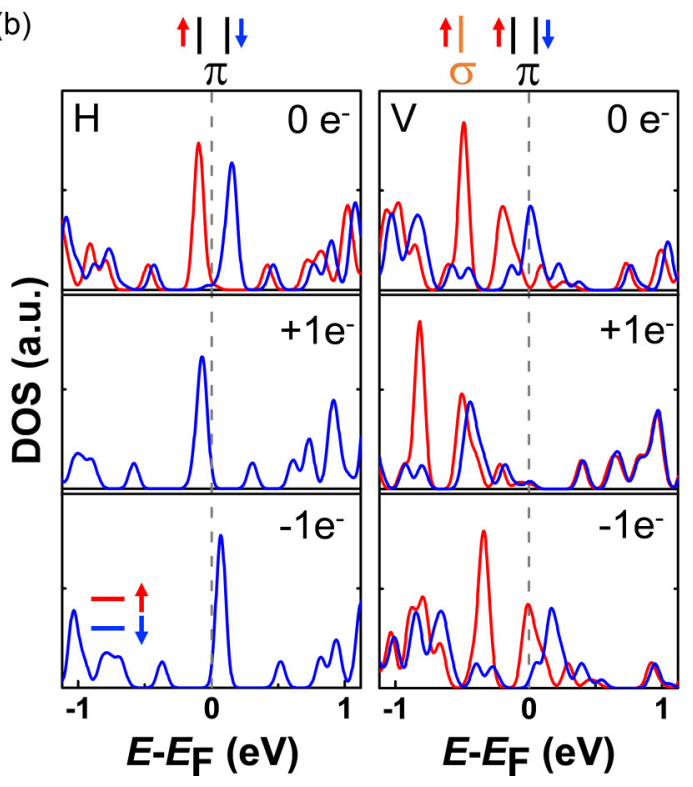

FIG. 1. Doping modulation in defects on graphene lattice. (a) Schematic diagram of a hydrogen adsorbate on graphene $(\mathrm{H})$ and a single-carbon vacancy (CV) in graphene. (b) Density of states of $\mathrm{H}$ and $\mathrm{CV}$ systems with and without doping. Red and blue lines represent spin-up and spin-down states, respectively. The sharp $\sigma$ peak in the right column locates on carbon atom 1, which is labeled in panel (a).

adsorption is $1 \mu_{\mathrm{B}}[28,29]$. Doping the $\mathrm{H}$ system by addition or removal of one electron destroys the spin-polarization through either double occupation $\left(+1 e^{-}\right)$or complete depletion of the $\pi$ band near the Fermi level $\left(E_{\mathrm{F}}\right)$, leading to full switch off of the magnetic moment [cf. left panel in Fig. 1(b). According to the Newns-Anderson-Grimley model, the local Coulomb interaction $U$ is responsible for the instability in the low-energy electronic states and lifts the spin-degeneracy of the $\pi$ states. [30]. Upon adding (removing) an electron, the $\mathrm{H}$ energy levels shift down (up) with respect to $E_{\mathrm{F}}$, and the splitting vanishes due to the transition from a magnetic state to a nonmagnetic state. Here, electrons and holes behave similarly due to the electron-hole symmetry around the Dirac point.

For the $\mathrm{CV}$, the magnetic moment originates from both the $\pi$ states near the $E_{\mathrm{F}}$ and a localized $\sigma$ dangling state, as shown in Fig. 1(b). Such a $\sigma$ state is mainly located on the apical carbon atom [C1 in Fig. 1(a)], leading to a sharp peak around $-0.6 \mathrm{eV}\left(0 e^{-}\right)$. The planar ground state of the undoped CV is ferromagnetic, and the wave functions of the $\sigma$ and the $\pi$ states are orthogonal in space [31-33]. This results in parallel spins of the two states according to Hund's rule. Moreover, the total moment is around $1.5 \mu_{\mathrm{B}}$ due to including the itinerant $\pi$ spins [34]. Addition of an electron to the $\mathrm{CV}$ increases the overall occupancy of the $\pi$ states (dominant peaks) such that they become doubly occupied leaving only one unpaired $\sigma$ state. In this case the total moment becomes $1 \mu_{\mathrm{B}}$. Similarly, removing one electron decreases the occupancy, yet the $\pi$ states are not fully unoccupied with a residual moment of $1.37 \mu_{\mathrm{B}}$. The reason for the different moments with hole versus electron doping can be attributed to an asymmetric splitting of the vacancy $\pi$ bands near $E_{\mathrm{F}}$. Importantly, unlike the $\mathrm{H}$ case, the spin splitting of the $\pi$ peaks can decrease with doping but cannot vanish, even if the two $\pi$ states are doubly occupied or unoccupied. The case of removing two electrons from the CV system is also checked, and there remains a little splitting of the $\pi$ bands (cf. Ref. [35], Fig. S1). These density-functional theory (DFT) results are in good agreement with the experimental findings $[11,13]$.

Graphene has a bipartite lattice [labels A and B shown as blue and red spheres, respectively, in Fig. 1(a)]. When a $\mathrm{H}$ or $\mathrm{CV}$ defect is created in the A sublattice, only $p_{z}$ orbitals of carbon atoms in the B sublattice contribute to the midgap or zero-energy $\pi$ state [36]. For the vacancy, in addition to the $\pi$ states, the vacancy $\sigma$ state is mainly located on the $\mathrm{C} 1$ atom, which also belongs to the B sublattice. The overlap between $\pi$ and $\sigma$ states hence determines the exchange splitting of the $\pi$ states near $E_{\mathrm{F}}$ in the vacancy. Even for double or empty occupation, the spin splitting of the $\pi$ defect state partially remains due to the exchange interaction with the polarized, unsaturated $\sigma$ states. Thus, the local magnetism of the $\mathrm{H}$ and $\mathrm{CV}$ systems are intrinsically different due to the contribution and persistence of the unsaturated $\sigma$ state in the CV.

Based on this understanding, how the voltage of a STM tip manipulates the local $\pi$ magnetism of the defects is further explored. We employ a three-terminal device setup for the transport calculations, encompassing left and right graphene electrodes, and an additional gold STM tip electrode, shown in Fig. 2(a). Generally speaking, both the electronic current and the field applied by the tip bias may affect the nonequilibrium transport process. In this regard, the tip-sample distance is essential because it determines the strength and distribution of the local field and furthermore influences the atomic and spin configuration directly by chemical interactions at close distance. Additionally, graphene devices often use an electrostatic backgate and also involve contact with a substrate, e.g., $\mathrm{SiC}$. Graphene is therefore typically doped by a gate and/or by the weak adsorption on surfaces [37-39] or by native impurities [40]. We model this by applying a gate plane placed $15 \AA$ underneath the graphene. The gate carries a charge density of $n=g \times 10^{13} e^{-1} / \mathrm{cm}^{2}$, where $g$ defines the gating level, with $g<0(g>0)$ corresponding to $n(p)$ doping [41]. In the following we focus on the control of the local spin states in the two scenarios by the tip potential and gate.

For the $\mathrm{H}$ atom on graphene, the smallest tip- $\mathrm{H}$ distance is $4.5 \AA$, where there is only a weak connection between the tip and H, shown in Ref. [35], Fig. S2. Starting from the ground state $\left(1 \mu_{\mathrm{B}}\right)$ at $0 \mathrm{~V}$, the calculated magnetic moment as a function of applied voltage is displayed in Fig. 2(b). Positive bias corresponds to positive graphene potential, see the inset in Fig. 2(a). With increasing positive bias voltage, the moment decreases and is completely switched off at $0.4 \mathrm{~V}$. 

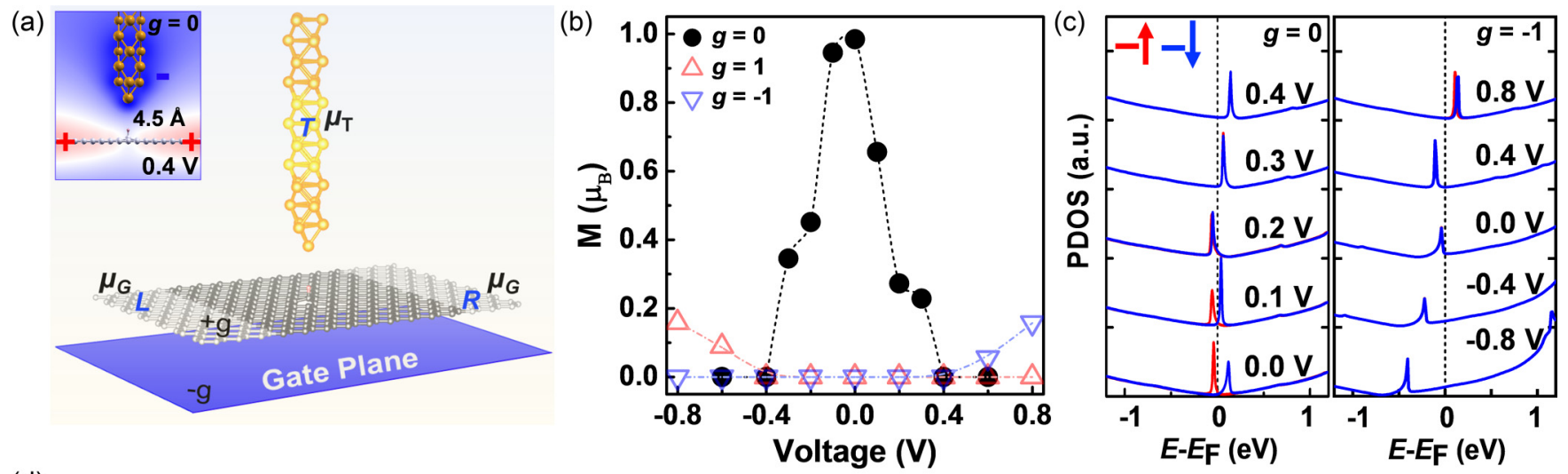

(d)

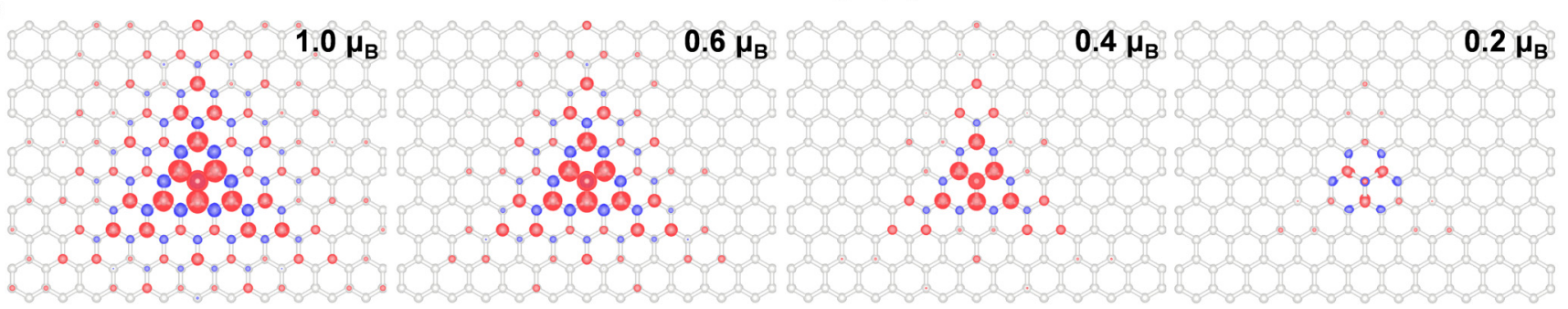

FIG. 2. Hydrogen adsorbate on graphene. (a) Schematic diagram of the three-terminal graphene device. The light gray and yellow spheres indicate left, right, and tip electrodes, respectively. Periodic boundary conditions are applied transverse to the left-right (L-R) direction in the graphene. The notation $\mu_{\mathrm{T}}$ and $\mu_{\mathrm{G}}$ stands for the chemical potential of the gold tip and graphene, respectively. The inset shows electrostatic potential profile at $0.4 \mathrm{~V}$ with $g=0$ gating. (b) Magnetic moment as a function of applied voltage. (c) Density of states projected onto the $p_{z}$ orbitals. Red and blue lines represent spin-up and spin-down states, respectively. (d) Spin-density distribution (isosurface values of $\left.\pm 0.005 e / \mathrm{bohr}^{3}\right)$, red and blue surfaces correspond to the densities of spin-up and spin-down states, respectively.

A negative bias can similarly tune the moment, because the splitting of the $\pi$ states at $0 \mathrm{~V}$ is symmetric near the $E_{\mathrm{F}}$. The calculated current is around $0.016 \mu_{\mathrm{A}}$ at $0.4 \mathrm{~V}$, which implies the mechanism here is governed by the tip-induced field or potential shift with little current effect. The tip acts as a local electrostatic gate, akin to local doping or gating and tunes the energy of the localized defect state. So for the positive bias, the H energy levels shift up with respect to the Fermi level, finally leading to an empty, degenerate spin-up and spin-down resonance at $0.4 \mathrm{~V}$ [cf. Fig. 2(c)]. In an electron-hole- and bias-symmetric manner, the resonances shift down, leading to completely filled, degenerate spin-up and spin-down levels for negative bias. The results show a transition from a magnetic state to a nonmagnetic state at the $\mathrm{H}$ atom on graphene can be realized by a moderate tip voltage, and the mechanism basically coincides with the simple picture of doping. The spin-density distribution in Fig. 2(d) roughly displays the spatial evolution of the magnetic moment from $1 \mu_{\mathrm{B}}$ to $0 \mu_{\mathrm{B}}$, corresponding to the tip voltage. For instance, the magnetic moment is $0.6 \mu_{\mathrm{B}}$ at the tip bias of $0.1 \mathrm{~V}$. Figure 2(d) also suggests the long-range magnetic interactions, in line with experimental observations [11].

For $n(p)$ doped with $g= \pm 1$ gating, the nonmagnetic state of $\mathrm{H}$ system at $0 \mathrm{~V}$ can be understood since $E_{\mathrm{F}}$ shifts up (down), as shown in Fig. 2(b). Due to the electron-hole symmetry, the spin behavior in the $n$ and $p$ cases is symmetric with respect to bias. In Fig. 2(c) we plot the density of states projected on the $\pi$ orbitals for $n$ doped with $g=-1$ gating. At $0 \mathrm{~V}$, a fully occupied $\pi$ peak is observed and continues to shift downwards for negative bias voltages without variation of the moment. When applying a positive bias, the $\pi$ band shifts up, and the change of its occupation gives rise to a moment at $0.6 \mathrm{~V}$ and a spin splitting of the $p_{z}$ peaks near $E_{\mathrm{F}}$. It means that the $\mathrm{H}$ atom on doped graphene can also be tuned from a nonmagnetic state to a magnetic state by a tip bias. Thus, it is possible to manipulate the spin states of doped graphene-based devices by either the local fields via a STM tip, or a global field from a gate electrode.

Next we consider the CV system where the presence of the unsaturated $\sigma$ bonds would affect the voltage control of the $\pi$ states, using a similar setup (see Ref. [35], Fig. S3). According to the results for charge doping, we note that it is not possible to completely suppress the vacancy magnetism by applying high voltages to empty or saturate the $\sigma$ band. Nevertheless, a metastable state (LS), only $15 \mathrm{meV}$ higher in energy than the ground state (HS), is associated with the vertical displacement of the carbon atoms near the vacancy, especially the height of the $\mathrm{C} 1$ atom $\left(h_{1}>0.3 \AA\right)$, cf. the inset in Fig. 3(a) $[34,42]$. Moreover, the bond length of the other two nearest-neighbor carbon atoms to the vacancy is $2.06 \AA$, a little larger than that in the HS state (1.99 $\AA$ ). This quantum LS state has a small magnetic moment of $0.5 \mu_{\mathrm{B}}$, due to the reversal of spin polarization of the $\pi$ bands near $E_{\mathrm{F}}[31,33,43]$, and can be formed and stabilized for intermediate tip-sample distances [20].

Figure 3(a) shows the calculated total-energy differences $\left(E_{\mathrm{diff}}=E_{\mathrm{HS}}-E_{\mathrm{LS}}\right)$ between HS and LS of monovacancy graphene with or without one-electron or one-hole doping. The atomic structures for two spin states here are fixed. The 


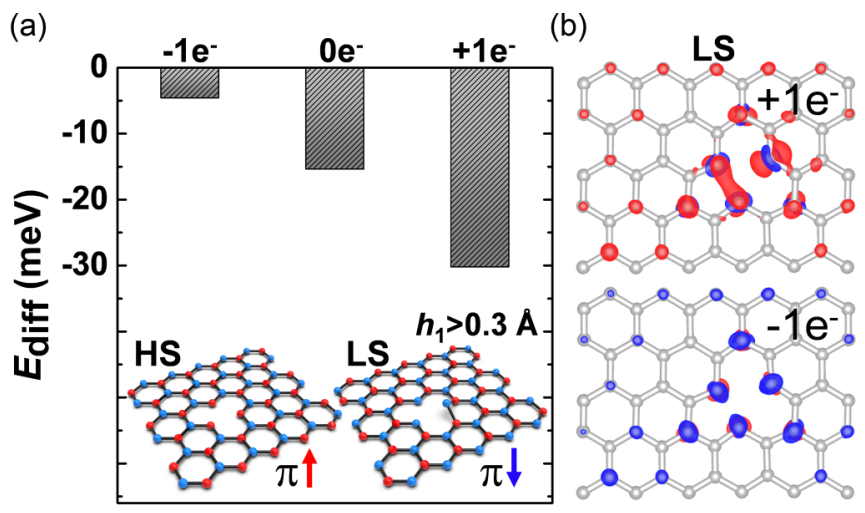

FIG. 3. The ground state, i.e., the high spin state (HS) and metastable state (LS) of monovacancy graphene. (a) Energy difference $\left(E_{\mathrm{diff}}=E_{\mathrm{HS}}-E_{\mathrm{LS}}\right)$ between HS and LS with and without doping. (b) Top view of charge-density difference after doping. Isosurfaces values of $\pm 0.001 \mathrm{e} / \mathrm{bohr}^{3}$ are shown. Red and blue clouds correspond to electron accumulation and depletion, respectively.

planar HS state remains as the ground state after doping, and $E_{\text {diff }}$ decreases from -4.6 to $-30.2 \mathrm{meV}$ as the number of electrons is increased. It has been found that, for the neutral cases $\left(0 e^{-}\right)$the energy barrier from HS to LS is $30.5 \mathrm{meV}$ and for the reverse process from LS to HS is $19.2 \mathrm{meV}$ [42]. This suggests that the HS state does not change by doping, but a spin switching from LS to HS would be more favorable after adding an electron in the nonplanar structure. The chargedensity difference for the LS state after electron or hole doping in Fig. 3(b) also supports this viewpoint: the local geometry would prefer to the planar structure with electron doping, but it prefers to remain in the out-of-plane geometry for hole doping. In addition, the carriers in all cases only exist on the graphene sublattice opposite to the one where the vacancy is created, implying the occupancy of the vacancy $\pi$ states is changed by doping. Therefore, the spin manipulation between HS and LS could be possible through a mechanism combining the variation of tip-defect distance and tip bias.

At zero bias, three magnetic states, HS, LS, and the nonmagnetic state (NS), can be realized as the Au tip approaches the $\mathrm{C} 1$ atom, corresponding to the three different tip-CV inter- action regimes, as shown in Fig. 4(a). The magnetic moments are $1.26 \mu_{\mathrm{B}}, 0.55 \mu_{\mathrm{B}}$, and $0 \mu_{\mathrm{B}}$, respectively. When the tip approaches the chemical interaction regime, the magnetism gets quenched by the strong coupling, implying that the electronic states of the tip and vacancy should be treated as a combined system. The system remains nonmagnetic, even when applying a high bias.

For the HS state, the tip-vacancy distance remains at $5 \AA$ in the transport calculations. As expected, the tip bias does not alter the local atomic vacancy structure on the $\pm 0.8 \mathrm{~V}$ scale. Like the $\mathrm{H}$ case, the tip also acts as a local gate. With increase of positive bias, the total moment increases at first and then decreases. This is caused by the upwards shift of the two $\pi$ resonances, where for small positive bias $(0.2 \mathrm{~V})$, the spin-down state loses more occupation than the spin-up state. For negative bias the $\pi$ peaks shift downwards, causing an increased filling of the spin-down state and a resulting decrease of the spin moment. The total moment of $1 \mu_{\mathrm{B}}$ is finally reached for both negative and positive bias when the vacancy $\pi$ states near the $E_{\mathrm{F}}$ become doubly unoccupied or occupied, with only the $\sigma$ band contribution. Despite the lack of a spin-flip transition for the $\mathrm{CV}$, the magnitude of the $\pi$ moment of the HS state can be tuned via the bias, promising for the STM manipulation.

In the LS case, the position of the vacancy energy levels depend both on the tip voltage and the tip-vacancy distance. Without bias, the distance between tip and graphene plane is $4.5 \AA$, and the vertical displacement of $\mathrm{C} 1$ is $0.72 \AA$. This results in the antiparallel spins of the $\sigma$ and $\pi$ electrons. Starting from it and increasing the positive bias, the moment first decreases slightly, and then increase to settle close to $0.8 \mu_{\mathrm{B}}$ at $0.6 \mathrm{~V}$, see Fig. 4(a). The two $\pi$ resonances shift up, and for small positive bias the spin-up state loses more occupation than the spin-down state. The reversal of spin polarization of the $\pi$ bands is responsible for this opposite trend, compared with the HS, cf. Fig. 4(b). When applying a small negative bias, the magnetic moment increases due to the downshift of the two $\pi$ levels. More importantly, according to the comprehensive picture of electron doping in the LS state in Fig. 3, the structural stability changes with higher applied negative voltage. A spin transition from the LS to HS states occurs at a bias of $-0.4 \mathrm{~V}$, corresponding to the atomic
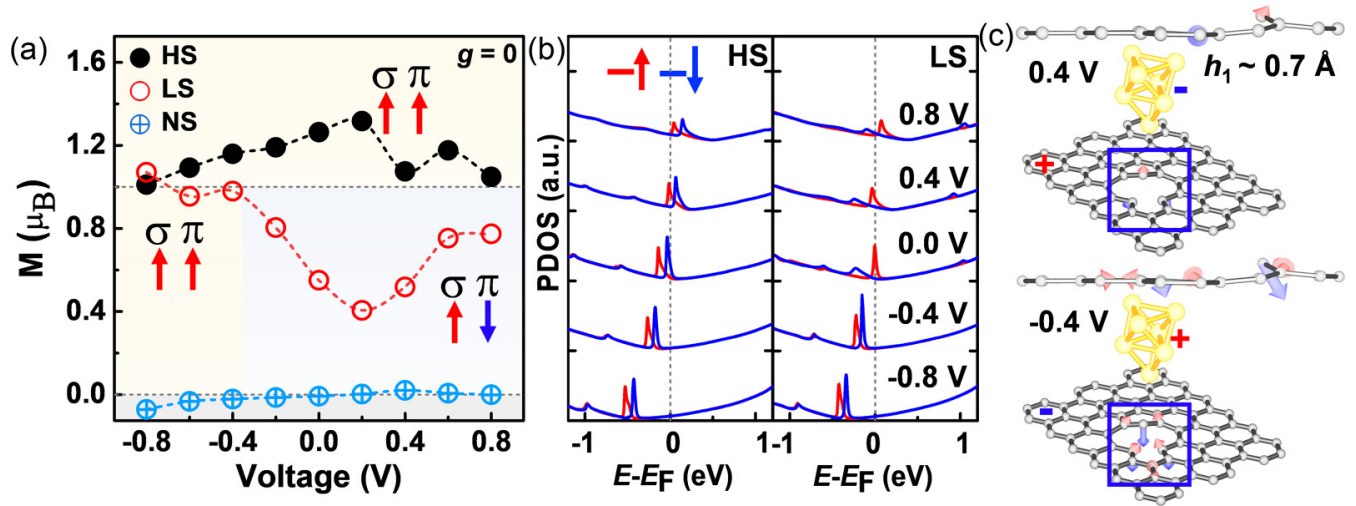

FIG. 4. Carbon vacancy. (a) The magnetic moment as a function of applied voltage for the three different vacancy spin states and conformations, HS, LS, and NS, respectively. (b) The density of states projected on the $p_{z}$ orbitals. The red and blue lines represent the spin-up and spin-down states. (c) Charge-induced force in the LS state at $\pm 0.4 \mathrm{~V}$. The absolute force on the $\mathrm{C} 1$ atom is $0.24 \mathrm{nN}$ at $-0.4 \mathrm{~V}$. 
geometry changing from a nonplanar structure to a nearly flat structure. Such a scenario is supported by the change in the electron density and atomic forces with bias. The chargedensity difference at $\pm 0.4 \mathrm{~V}$ is almost the same as that of the LS state after removing or adding an electron, respectively, as shown in Fig. 3(b). Moreover, the charge-induced forces on the carbon atoms close to the vacancy suggest that it remains an out-of-plane structure at $0.4 \mathrm{~V}$ and favors a planar structure at $-0.4 \mathrm{~V}$, as shown in Fig. 4(c). The absolute force of 0.24 $\mathrm{nN}$ is exerted on the $\mathrm{C} 1$ atom at $-0.4 \mathrm{~V}$, which drives the $\mathrm{C} 1$ atom down to the graphene plane. These forces are calculated in the Born-Oppenheimer approximation from the nonequilibrium electron density [23], and can be compared directly with experiments [44,45]. Our spin-transport calculations thus suggest that the two vacancy spin states, HS and LS, can be manipulated reversibly by a coordination mechanism between an intermediate tip-defect distance and a moderate tip voltage. In an earlier work, a two-terminal graphene CV device showed that the $\pi$ states can be modified by an in-plane bias voltage, however, only to a little degree [19]. It indicates that it is hard to manipulate the vacancy states without a tip or gating.

In conclusion, we have performed first-principles DFTnon-equilibrium Green's function calculations to study the manipulation of the localized $\pi$ magnetism at two types of defects in graphene by using a STM tip. It is found that the magnetism of the $\pi$ states strongly depend on both the tip voltage and the tip-defect distance. In the $\mathrm{H}$ case, the magnetic moment can be switched on and off with a tip voltage or a gate plane. For the carbon vacancy, the repulsive Coulomb interaction between the unsaturated $\sigma$ and $\pi$ electrons is responsible for the stable spin splitting of the $\pi$ bands around the Fermi level. Importantly, a spin switching from the LS to HS states in CV system can be achieved by tuning the tip-vacancy distance and the tip bias. The issues on possible long length scale and long timescale magnetic order are also important and will be pursued in future investigations. Our findings support the prospect of the local control of graphene magnetism on the atomic scale. We believe that it is versatile and could find potential applications in spintronics and magnetic sensing.

This project received funding from the EU Horizon 2020 under Grant No. 766726. C.N.G. is sponsored by the Danish Research Foundation (DNRF103). S.G. acknowledges support from the National Natural Science Foundation of China (11934003, 21961132023, U1930402) and the MOST of China (2017YFA0303404).
[1] W. Han, R. K. Kawakami, M. Gmitra, and J. Fabian, Nat. Nanotechnol. 9, 794 (2014).

[2] Z. Wang, T. Zhang, M. Ding, B. Dong, Y. Li, M. Chen, X. Li, J. Huang, H. Wang, X. Zhao et al., Nat. Nanotechnol. 13, 554 (2018).

[3] N. Tombros, C. Jozsa, M. Popinciuc, H. T. Jonkman, and B. J. van Wees, Nature (London) 448, 571 (2007).

[4] X. Yu, H. Cheng, M. Zhang, Y. Zhao, L. Qu, and G. Shi, Nat. Rev. Mater. 2, 17046 (2017).

[5] S. Jiang, J. Shan, and K. F. Mak, Nat. Mater. 17, 406 (2018).

[6] A. Avsar, H. Ochoa, F. Guinea, B. Özyilmaz, B. J. van Wees, and I. J. Vera-Marun, Rev. Mod. Phys. 92, 021003 (2020).

[7] K. S. Novoselov, A. K. Geim, S. V. Morozov, D. Jiang, Y. Zhang, S. V. Dubonos, I. V. Grigorieva, and A. A. Firsov, Science 306, 666 (2004).

[8] K. S. Novoselov, A. K. Geim, S. V. Morozov, D. Jiang, M. I. Katsnelson, I. Grigorieva, S. Dubonos, and A. Firsov, Nature (London) 438, 197 (2005).

[9] V. N. Kotov, B. Uchoa, V. M. Pereira, F. Guinea, and A. H. Castro Neto, Rev. Mod. Phys. 84, 1067 (2012).

[10] J. Tuček, P. Błoński, J. Ugolotti, A. K. Swain, T. Enoki, and R. Zbořil, Chem. Soc. Rev. 47, 3899 (2018).

[11] H. González-Herrero, J. M. Gómez-Rodríguez, P. Mallet, M. Moaied, J. J. Palacios, C. Salgado, M. M. Ugeda, J.-Y. Veuillen, F. Yndurain, and I. Brihuega, Science 352, 437 (2016).

[12] M. M. Ugeda, I. Brihuega, F. Guinea, and J. M. GómezRodríguez, Phys. Rev. Lett. 104, 096804 (2010).

[13] Y. Zhang, S.-Y. Li, H. Huang, W.-T. Li, J.-B. Qiao, W.-X. Wang, L.-J. Yin, K.-K. Bai, W. Duan, and L. He, Phys. Rev. Lett. 117, 166801 (2016).

[14] J. Fernández-Rossier and J. J. Palacios, Phys. Rev. Lett. 99, 177204 (2007).
[15] O. V. Yazyev, Phys. Rev. Lett. 101, 037203 (2008).

[16] W. L. Wang, O. V. Yazyev, S. Meng, and E. Kaxiras, Phys. Rev. Lett. 102, 157201 (2009).

[17] E. H. Lieb, Phys. Rev. Lett. 62, 1201 (1989).

[18] F. Gao and S. Gao, Sci. Rep. 7, 1792 (2017).

[19] S. Wen, F. Gao, C. Yam, and S. Gao, Carbon 133, 218 (2018).

[20] Y. Zhang, F. Gao, S. Gao, and L. He, Sci. Bull. 65, 194 (2020).

[21] J. P. Perdew, K. Burke, and M. Ernzerhof, Phys. Rev. Lett. 77, 3865 (1996).

[22] J. Klimeš, D. R. Bowler, and A. Michaelides, J. Phys.: Condens. Matter 22, 022201 (2009).

[23] M. Brandbyge, J.-L. Mozos, P. Ordejón, J. Taylor, and K. Stokbro, Phys. Rev. B 65, 165401 (2002).

[24] J. M. Soler, E. Artacho, J. D. Gale, A. García, J. Junquera, P. Ordejón, and D. Sánchez-Portal, J. Phys.: Condens. Matter 14, 2745 (2002).

[25] N. Papior, N. Lorente, T. Frederiksen, A. García, and M. Brandbyge, Comput. Phys. Commun. 212, 8 (2017).

[26] G. Kresse and J. Hafner, Phys. Rev. B 47, 558 (1993).

[27] N. Papior, https://doi.org/10.5281/zenodo.597181, sisl, v0.10.0 (2020).

[28] O. V. Yazyev and L. Helm, Phys. Rev. B 75, 125408 (2007).

[29] D. W. Boukhvalov, M. I. Katsnelson, and A. I. Lichtenstein, Phys. Rev. B 77, 035427 (2008).

[30] D. M. Newns, Phys. Rev. 178, 1123 (1969).

[31] P. O. Lehtinen, A. S. Foster, Y. Ma, A. V. Krasheninnikov, and R. M. Nieminen, Phys. Rev. Lett. 93, 187202 (2004).

[32] J. J. Palacios and F. Ynduráin, Phys. Rev. B 85, 245443 (2012).

[33] A. A. El-Barbary, R. H. Telling, C. P. Ewels, M. I. Heggie, and P. R. Briddon, Phys. Rev. B 68, 144107 (2003).

[34] H. Padmanabhan and B. R. K. Nanda, Phys. Rev. B 93, 165403 (2016). 
[35] See Supplemental Material at http://link.aps.org/supplemental/ 10.1103/PhysRevB.103.L241402 for calculated projected densities of states of monovacancy graphene with and without p-doping (Fig. S1), tip-defect distance in the $\mathrm{H}$ case (Fig. S2), three-terminal device setup for monovacancy graphene (Fig. S3) and charge-density difference and charge induced force in the LS state of monovacancy graphene at $\pm 0.4 \mathrm{~V}$ (Fig. S4).

[36] B. Nanda, M. Sherafati, Z. S. Popović, and S. Satpathy, New J. Phys. 14, 083004 (2012).

[37] P. A. Khomyakov, G. Giovannetti, P. C. Rusu, G. Brocks, J. van den Brink, and P. J. Kelly, Phys. Rev. B 79, 195425 (2009).

[38] G. Giovannetti, P. A. Khomyakov, G. Brocks, V. M. Karpan, J. van den Brink, and P. J. Kelly, Phys. Rev. Lett. 101, 026803 (2008).
[39] C. Coletti, C. Riedl, D. S. Lee, B. Krauss, L. Patthey, K. von Klitzing, J. H. Smet, and U. Starke, Phys. Rev. B 81, 235401 (2010).

[40] R. R. Nair, I.-L. Tsai, M. Sepioni, O. Lehtinen, J. Keinonen, A. V. Krasheninnikov, A. H. Castro Neto, M. I. Katsnelson, A. K. Geim, and I. V. Grigorieva, Nat. Commun. 4, 2010 (2013).

[41] N. Papior, T. Gunst, D. Stradi, and M. Brandbyge, Phys. Chem. Chem. Phys. 18, 1025 (2016).

[42] F. Gao and S. Gao, Sci. Rep. 9, 751 (2019).

[43] C.-C. Lee, Y. Yamada-Takamura, T. Ozaki et al., Phys. Rev. B 90, 014401 (2014).

[44] J. Brand, S. Leitherer, N. R. Papior, N. Néel, Y. Lei, M. Brandbyge, and J. Kröger, Nano Lett. 19, 7845 (2019).

[45] M. Omidian, S. Leitherer, N. Néel, M. Brandbyge, and J. Kröger, Phys. Rev. Lett. 126, 216801 (2021). 